\title{
Fully Integrated Hemodynamic Interventional Laboratory Informative System
}

\author{
A Mazzarisi, P Marraccini, P Marcheschi, G Djukic, R Favilla, I Marinaro, M Dalle Luche, \\ A Benassi
}

\author{
CNR Institute of Clinical Physiology, Pisa, Italy
}

\begin{abstract}
An invasive cardiology department produces an intense and heterogeneous information flow. Our Institute manages all the activities yearly performed (700 diagnostic and 300 interventional procedures) through a large database allowing a real time tracing of the work process features (waiting list, procedure protocols, medical reports, images file, administrative and organizational procedures). A selected information subset, automatically transferred from the hEmodyinamic Information System (SIE) to the central database of the institute (ARCA), receives data from in and out patient departments, laboratories and medical diagnostic technique image departments, and feeds the electronic patients record. SIE, which lays on multiplatform and open source products, makes clinical work easier by organizing information data flow and data viewing, improves the interpretation of clinical data, reduces the decision-making process and increases operational efficiency.
\end{abstract}

\section{Introduction}

Managing a modern invasive cardiological department requires the optimization of heterogeneous informative data flows, which can either be records generated from administrative and clinical departments or multimedia format data produced through net systems [1].

The SIE system, operative since 1997 and continuously updated, has been conceived as a tool for clinical activities, to evaluate clinical protocols and develop research studies applied in the field of invasive cardiology. For this reason SIE is a custom-made system, quickly adaptable to exchange data with analogical and digital instrumentation. In particular, the aims pursued for the planning of this system are:

1) To develop methodologies and tools for the gathering of specific data from invasive cardiological laboratories, both by the employment of appropriate equipments and software, and regarding some parameters (performances, costs, scalability, heterogeneity of use on different platforms, open source solutions to guarantee interoperability and standardization);

2) To allow collection, presentation and storage of information in relation to events and clinical needs, so to ease the interpretation of the events and therefore the decision making process;

3) To improve service quality through authorized and automatic quality control procedures.

\section{Methods}

The technical requirements of SIE have been settled to:

1. Work on different operating rooms and groups contemporarily;

2. Make use of the joint activity of all the professional figures operating in an invasive cardiological laboratory;

3. Manage waiting list and procedure booking according to the centralized service, or in autonomous way when appropriate;

4. Prepare both the daily work program for medical operators and the diagnostic equipments through the DICOM Worklist [3].

5. Give on-line help for a correct use of layouts during the work process, for each professional category (administratives, technicians, nurses and physicians);

6. Provide a semiautomatic support to fill in diagnostic and interventional reports;

7. Supply on-line reports and images through Web technologies, both on Local Area Network (LAN) and on Wide Area Network (WAN - Internet).

In addition, it has been required the use of modern data integration technologies with external computer systems (SQL, ODBC, JDBC, SERVLET JAVA, XML, XLST, DTD), and the resort to diversified access profiles on the basis of role and specific user.

To improve service quality it has been necessary the use of automatic indicators to monitor activity and give information about events and use of resources (materials and personnel).

All the software and hardware tools have been realized by the CNR Institute of Clinical Physiology computer team.

The structured procedures regarding clinical and organizational workflow have been implemented via multi-vendor platforms such as Windows, Mac OS 9 and Mac OS X, which give a support to medical operators for the activity planning, to manage single clinical case and 
materials, and to evaluate procedural costs.

Images and data archive servers (image sequences and clinical administrative and managerial data) have been installed on the consumer platform and with redundant configurations.

In our laboratory some specific rules for data management and treatment have been adopted:

1. The image archive server keeps about one year storage and has to be used in DICOM standard;

2. The archiving of clinical data must be strongly structured;

3. Specific procedures to guarantee privacy, data archiving security, crash and disaster recovery must be followed.

\section{System overview}

SIE is used in a catheterization laboratory organized with two operative rooms.

The Staff consists of five physicians, ten nurses, three radiology technicians, two administrative people and two software technicians.

The Software provides the following services:

1. Activity planning: it consists of procedures made for the waiting list management and the daily work planning, including patient's list, medical operator and nursing activities for each catheterization laboratory; anamnestic, clinical and administrative data retrieving procedures from the centralized Electronic Patient Record (EPR) [2], and other informative services when allowed.

2. Data storage and workflow relationship: the data storage is driven by a workflow process allowing to keep temporal hierarchic traces of data. According to this rule, the records collected comprise procedural and post-procedural data ( 6 and 24 hours after examination), daily monitoring of instrumentations and stock device management.

3. On-Line report services: report and image diagnoses supply EPR and authorized clients from other external laboratories:

- Medical reports and clinical comments;

- Pre and post procedural scores useful for quality control procedures;

- Iconographic documents, including standard elaboration and images annotation;

- Teleconsulting, consisting in the transmission of full image angiographic sequences and in the sharing of information through DICOM format in LAN and WAN.

4. Device Stock Management: an autonomous sub system, integrated with SIE, manages all medical disposable instrumentation during daily activity.

The Hardware employed is the following:

1. Management and storage data servers using Linux and FileMaker Pro as Relational databases, able to run up to 50 simultaneous users;

2. Management and storage of angiographic images and sequence servers, using Linux and an Open Source "DCM4CE" DICOM server with a 1000 Giga Byte disk array;

3. Worklist services, EPR's Referral Images Broker and a Teleconsulting DICOM Broker, using workstations with Open Source "DCMTK" DICOM library;

4. Query and filling data on central database services, using a JAVA 1.4 standard hardware machines, with Apache and the Tomcat framework.

\section{Basic security}

SIE has realized an important collection of data that could enrich knowledge in the Hemodynamic field, thus it requires the performance of complex activities for its maintenance and to guarantee the privacy of patient's information and data:

1. Data Access and Security: physical access to SIE and ARCA resources is made with the adoption of two Firewall, while SSH and security digital certifies are necessary with intranet and Internet image's and data consultation. User and Password managed by SIE is required to have access to every software tool and to identify the work profile of each user.

2. Data Integrity: Raid Systems for Data and Image Archive Server, together with daily backup policies, have been adopted.

3. Disaster Recovery: a double hardware guarantees SIE from crash and allows a fast disaster recovery procedure. Moreover, such architectures allow the testing of new functionalities without any stop of SIE.

\section{HIS integration}

\subsection{Internal and external data flow}

The central database of CNR Institute of Clinical Physiology, named ARCA (IBM DB2 and IBM AIX operative system), receives information from different data sources (in and out patient departments, clinical laboratories and administrative services) and organizes the EPR. SIE (figure 1) through calls to a servlet JAVA and interpretation of XML documents has access to each table of ARCA database [4,5]. Thus SIE can retrieve patient's data from EPR. Such retrieval functions are activated, for each patient, at the time of the access in the waiting list and at the beginning of the invasive procedure. At the end of each invasive procedure SIE sends to ARCA a list of selected data. In addition, key angiographic images are selected by the physician from the report workstation and sent to the local temporary storage DICOM SCP (Service Class Provider). These multimedia data are sent to ARCA, using time scheduled routines written in JAVA, that have an access to specific fields of EPR. At the end of this cycle ARCA allows the consultation of reports, images and annotations to registered clients via WEB based techniques. 


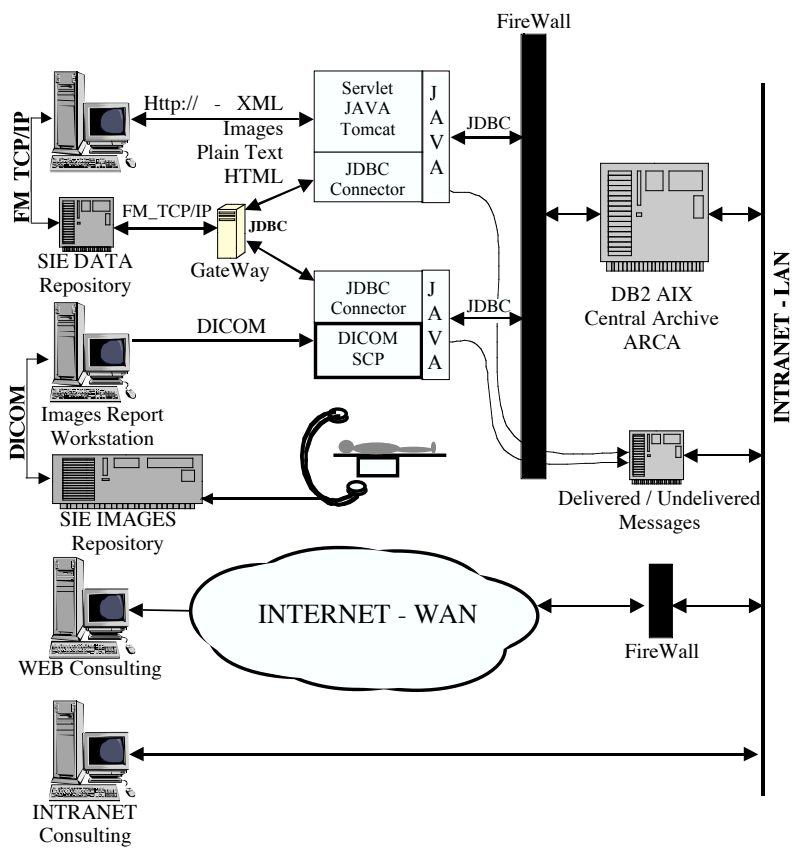

Figure 1. Hemodynamic Interventional Lab Architecture

\subsection{Nurses annotation}

Information regarding kind of procedure, possible complications, drugs administration, nursing activities during invasive examinations, and suggestion for the postprocedural assistance, are organized in a structured document. Such document is automatically generated and sent to the nursery database of the cardiological department (GIR). SIE is integrated with GIR via a message broker that is independent from the two systems.

\subsection{Work list supply}

At the beginning of each invasive examination SIE allows to generate a specific structured data containing key information to identify the procedure (ID number, first and family name, date of birth, height, weight), according to the rules of the Performed Procedure Step Protocol of DICOM standard. The WORKLIST server, built on open source DCM4CE libraries, supplies these data to the radiological machines.

\section{Key actions}

\subsection{In-hospital follow-up}

Such tool has been introduced to improve care quality after an invasive examination. In fact, post-procedural complications might be relevant from a clinical and a prognostic point of view, but they are frequently overlooked by the catheterization laboratory staff. The monitoring of post-procedural complications allows a precise analysis of these events and may be useful to improve surveillance protocols and patient care. Every 6 and 24 hours after the examinations nurses collect data concerning the following types of events: cardiac (recurrence of angina, ECG patterns), neurological (consciousness, sensitivity and strength), renal (diuresis), local (hematoma, arterial pulse and limb temperature). The taken information are checked by a physician, who can dispose the proper treatment.

\subsection{WEB sharing resource}

SIE clients are both physicians operating into the CNR Institute of Clinical Physiology and physicians working in external cardiological departments and referring patients to the invasive laboratory. Clients may consult SIE archive by a standard Internet browser (Figure 2). The access is allowed according to the client's role and function by an authentication protocol (HTTPS with use of authenticity certificate). For example, physicians from external departments may consult only the information related to their referred patients, with given informed consent to data treatment. This service was well accepted by external physicians, because it has improved their participation in the clinical decision making and it has allowed a continuous self-governing consultation of patient data.

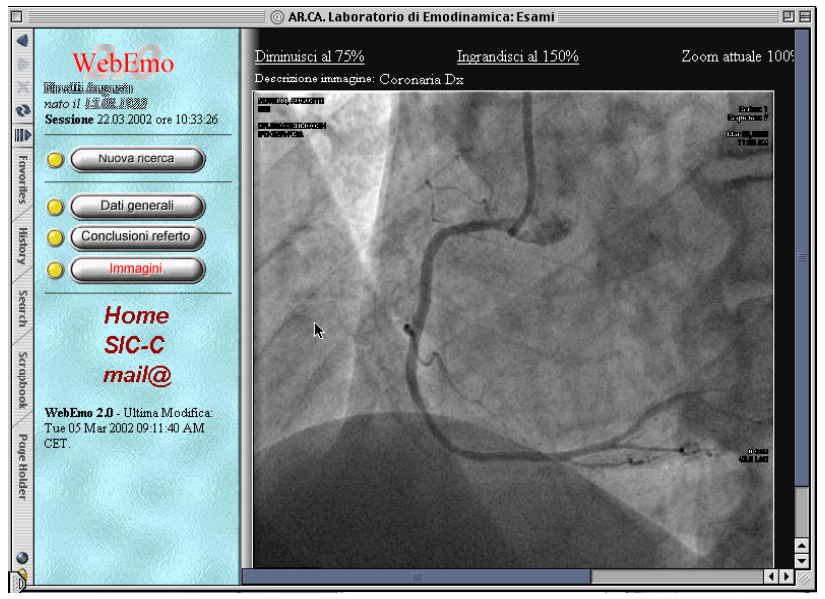

Figure 2. WEB consulting

\subsection{Teleconsulting facility}

An effective collaboration between cardiological department, invasive cardiological and cardio-surgical departments is based on a real share of information and on the possibility to take part to meetings and consultations. This is particularly crucial in our Institute, because the cardio-surgical department is not located in the same area. Cardiology and cardio-surgical departments are linked by a net connection with links at $2 \mathrm{Mbit} / \mathrm{s}$ and $256 \mathrm{Kbit} / \mathrm{s}$ (backup ISDN). A teleconsulting system operating on the above mentioned net has been activated (Figure 3); it is able to manage information from SIE and EPR and, in addition, to exchange multimedia documents including images and complete movie sequences related to patient examinations in DICOM standard. Data is encoded by DICOM TLS and transferred using a TCP/IP protocol. 
This transfer service has been named "DICOM Broker" and allows all the operations for the distribution and the management of images and movies in DICOM format. Moreover, commercial instruments for video conferencing (Net Meeting, CuSeeMe) have been included in this service.

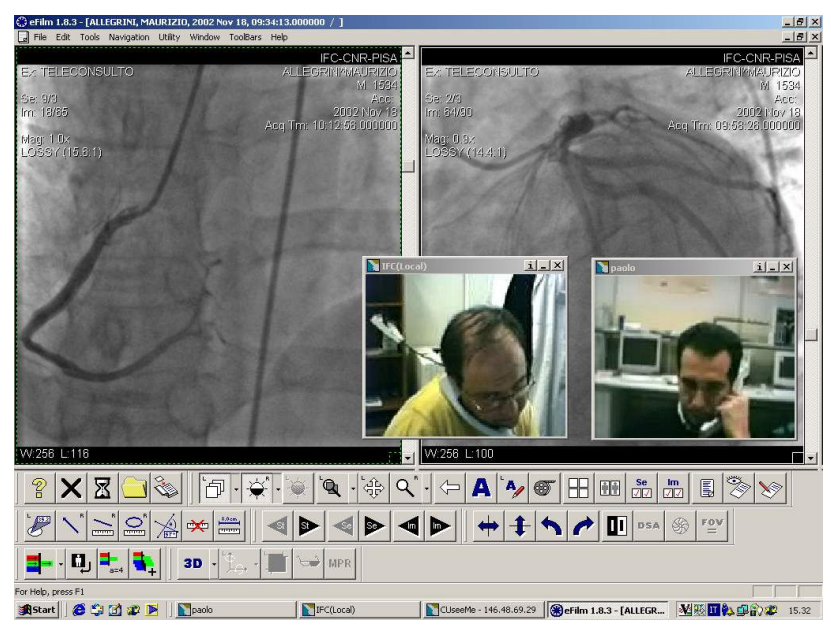

Figure 3. Teleconsulting session with DICOM Viewer

\subsection{Device stock management}

The availability of specialized devices is crucial for the daily activity of a modern invasive cardiological laboratory. The number and range of such products is very large and, moreover, biomedical companies propose continuous updates and innovations. The majority of such devices are disposable and have a deadline limiting their usage in time. Their cost is very high, so those represent an important item in the balance sheet of an invasive cardiology department. For this reason, a very important part of SIE is deputed to monitor the use of such devices, in order to organize statistical evaluations allowing a rational stock of this biomedical material. The type and amount of devices used in each invasive procedure is unloaded on-line by a bar-code system. The same system is also used to load the material incoming from an administrative consol. A strict control of in and out coming flow of products is assigned to two qualified operators.

This informative system has allowed to manage the device stock according to the weekly consumption and to automatically generate orders through telematic tools.

\section{Conclusion}

The SIE described in this document is an example of open source tools and multiplatform software application, able to manage an activity, as complex as an invasive cardiological department. SIE is applied either to manage health care routine activities, to test new clinical protocols of to follow research projects. SIE is in continuous evolution, moreover its complexity and importance grows also in relation with other informative systems.

The integration realized through exchanges of highlevel messages based on standard protocols like HL7 and DICOM, it will provide in the future, the most general answer for all the environments, where are present multivendors installations. Today this kind of solution is present only in small and averages sanitary structures of our country.

\section{Acknowledgements}

The authors are particularly grateful to external cardio logical departments (Prof. Francesco Marchi H Santa Maria Nuova Firenze, Dr. Daniele Bernardi H Castelnuovo Lucca and to Dr. Silva Severi H Grosseto) that collaborated to test WEB resources. Moreover, we acknowledge Dr. Elisa Marsigli for the secretarial assistance in the preparation of this manuscript.

\section{References}

[1] Taddei A, Carpeggiani C et al. Development of electronical medical record for patient care in cardiology. Computers in Cardiology 1997; 24:641-644.

[2] Taddei A, Macerata A, Dalmiani S et al. Medical record system for cardiology and cardiac surgery. Computers in Cardiology 1999;26:85-88

[3] DICOM Standards Committee. The DICOM Standard. http://medical.nema.org/dicom/2003.html

[4] SUN Microsystems. The Source for Java Tecnology. http://java.sun.com/

[5] W3C. Extensible Markup Language (XML). http://www.w3c.org/XML/

Address for correspondence:

Alessandro Mazzarisi

CNR Institute of Clinical Physiology

CNR Research Area - Via G.Moruzzi 1

56124 PISA (ITALY)

E-mail address: mazzaris@iff.cnr.it 